\title{
190 Pathology of allergic rhinitis
}

A Oedematous swelling of the mucosa occurs.

B There is infiltration with giant epithelioid cells.

C Seromucinous glands atrophy.

D Venous stasis results in a dusky swelling of the inferior turbinates.

E Polyp formation is a rare complication.

\section{Clinical features of allergic rhinitis}

A Symptoms may be seasonal or perennial.

B Age of onset is usually in the fourth decade.

C Anosmia is the main complaint.

D Sneezing attacks may be incapacitating.

E Polyps should be suspected if nasal obstruction is permanent.

\section{Treatment of allergic rhinitis}

A Avoidance of allergens is frequently impractical.

B Desensitization based on skin testing is useful in some cases of hay fever.

C Vasoconstrictor drops provide effective immediate relief.

D Sedation is the major side effect of antihistamines.

E Local steroids and sodium cromoglycate both act to reduce the hypersensitivity reaction.

\section{Surgical treatment of allergic rhinitis}

A is preferable to long term medication in children.

B Is indicated where chronic nasal obstruction is due to polyps.

C Adenoidectomy is the first line of treatment in children.

D Reduction of inferior turbinates is indicated for sneezing.

E Vidian neurectomy can be performed by a transantral approach. 